\title{
A NOVEL MULTIPLE CRITERIA DECISION-MAKING METHOD USING A LIKELIHOOD-BASED COMPROMISE APPROACH UNDER COMPLEX UNCERTAINTY
}

\author{
Jih-Chang Wang ${ }^{1}$, Ting-Yu Chen ${ }^{2}$ \\ ${ }^{1}$ Assistant Professor, Department of Information Management, Chang Gung University, Taoyuan, Taiwan \\ ${ }^{2}$ Professor, Graduate Institute of Business and Management, Chang Gung University, Taoyuan, Taiwan \\ ${ }^{3}$ Adjunct Research Fellow, Division of Cerebrovascular Disease, Department of Neurology, Linkou Chang Gung \\ Memorial Hospital, Taoyuan, Taiwan
}

\begin{abstract}
The purpose of this paper is to develop a new multiple criteria decision analysis method that uses a likelihood-based compromise approachwithin the environment of interval type-2 fuzzy sets. The theory of interval type-2 fuzzy sets, interval type-2 trapezoidal fuzzy numbers especially, is useful and valuable for modeling impressions and quantifying the ambiguous nature of subjective judgments. This paper presents some novel concepts of likelihood indices, weighted likelihood indices, likelihood-based compromise indices, and likelihood-based compromise values. Using a likelihood-based compromise approach, this paper develops a simple and effective method that can address the multiple criteria decision-making problems and avoid the computational complexity attached to interval type-2 trapezoidal fuzzy numbers. This paper introduces the concept of approximate positive-ideal and negative-ideal solutions to facilitate anchored judgments about the evaluative ratings of alternatives. Based on the likelihood of a binary relation, this paper establishes the likelihood index and the weighted likelihood index relative to the approximate ideals. A novel likelihood-based compromise index of the characteristics for each alternative is proposed to determine the corresponding likelihood-based compromise value using a signed distance-based method. This paper develops an algorithmic procedure using the proposed likelihood-based compromise approach for the purpose of acquiring the priority orders of the alternatives. Finally, the feasibility and applicability of the proposed method are illustrated with a practical application.
\end{abstract}

Keywords: Multiple criteria decision analysis, Interval type-2 fuzzy set, Likelihood index, Approximate ideal, Likelihood-based compromise index.

\section{INTRODUCTION}

Multiple criteria decision analysis (MCDA) often takes place within a complex and uncertain environment and involve conflicting systems of criteria (Chen, 2017; Qin et al., 2017). Considering the decision maker's point of view and circumstances in the decision-making process, subjective opinions and judgments are inherently imprecise and involve many uncertainties (Chen, 2014a;Singh and Garg, 2017). In fuzzy community, Zadeh (1975) introduced fuzzy sets of Type-2, later shortened by others to type-2 fuzzy sets (T2 FSs) and fuzzy sets with interval-valued membership functions, which was later shortened by others to interval-valued fuzzy sets (Mendel, 2007; 2010). The concept of T2 FSs is an extension of type-1 fuzzy sets (T1 FSs) and is characterized by a fuzzy membership function, where the degree of membership for any element in this set is a fuzzy number in the interval $[0,1]$ (Chen, 2013; 2017; Zhou et al., 2017). T2 FSs are better than T1 FSs for handling imprecision and uncertainties by modeling vagueness and unreliability of information (Chen, 2017; Zhou et al., 2017). Real-world decisions always require the use of more precise and accurate data (Lai and Chen, 2015); thus, T2 FSs are appropriate for addressing real-life problems if there is insufficient knowledge or experience (Chen, 2014a; 2014b; Zhang and Zhang, 2013).

However, the membership function of T2 FSs is usually difficult and troublesome to determine, which hinder their practical applications (Siminski, 2017). Resolving the difficulties in establishing and handling the secondary membership functions, interval type-2 fuzzy sets (IT2 FSs) are the most widely used of the higher order fuzzy sets because of their relative simplicity (Mendel, 2007; Siminski, 2017; Wu and Mendel, 2007). IT2 FSs are a simplified version of T2 FSs, and the membership grades of IT2 FSs are crisp intervals (Siminski, 2017; Wang et al., 2015). The theory of IT2 FSs has been well developed in the literature and has been applied productively in the field related to MCDA under uncertainty (Celik and Taskin Gumus, 2016; Chen, 2014a; 2014b; Lai and Chen, 2015; Singh and Garg, 2017; Wang and Chen, 2014; Wang et al., 2015; Zhang and Zhang, 2013; Zhong and Yao, 2017; Zhou et al., 2017). In particular, interval type-2 trapezoidal fuzzy numbers (IT2 TrFNs), as a special case of IT2 FSs, can efficiently express linguistic ratings and evaluations by objectively transforming them into numerical variables (Chen, 2017; Zhang and Zhang, 2013). 
Based on the decision context of IT2 TrFNs, the purpose of this paper is to develop a new MCDA method that uses a likelihood-based compromise approachwithin the environment of IT2 FSs. This paper presents the concept of approximate positive-ideal and negative-ideal solutions to make anchored judgments concerning the IT2 TrFN evaluative ratings of alternatives. A specific alternative performs well with respect to benefit criteria when its IT2 TrFN evaluative ratings have a low possibility of being inferior to the ratings of the approximate positive-ideal solution and a high possibility of being superior to the ratings of the approximate negative-ideal solution. On the other hand, an alternative performs well in terms of cost criteria when its IT2 TrFN evaluative ratings have a low possibility of being farther from the ratings of the approximate positive-ideal solution and a high possibility of being farther from the ratings of the approximate negativeideal solution. However, for example, any alternative which has the lowest possibility of being inferior to the approximate positive-ideal solution is not guaranteed to have the highest possibility of being superior to the approximate negativeideal solution. With these considerations, this paper proposes a likelihoodbased compromise approach to facilitate determination of the priority order of alternative actions.

This paper employs the extended concept of likelihoods of IT2 TrFN binary relations to construct the likelihood index and the weighted likelihood index relative to the approximate positive-ideal and negative-ideal solutions. Additionally, this paper establishes the likelihood-based compromise index and its corresponding compromise value for the purpose of acquiring the priority orders of the alternatives. The feasibility and applicability of the proposed method are illustrated using the car evaluation problem. In contrast to existing MCDA methods within the IT2 FS environment, the proposed MCDA method using a likelihood-based compromise approach is simple and easy to implement. Furthermore, the proposed method provides a convenient and computationally feasible way of dealing with uncertain and ambiguous properties in MCDA problems. Based on the developed likelihood indices and likelihood-based compromise indices, the proposed method can effectively address the MCDA problems based on IT2 TrFNs and to avoid the computational complexity attached to IT2 FSs.

The remainder of this paper is organized as follows. Section 2 briefly reviews the concepts of IT2 FSs and IT2 TrFNs. Section 3 formulates a decision environment based on IT2 TrFNs and develops an MCDA method using a likelihoodbased compromise approach. Section 4 demonstrates the feasibility and the applicability of the proposed method using a practical application of the car evaluation problem. Finally, Section 5 presents the conclusions.

\section{PRELIMINARIES}

This section reviews some relevant definitions and operations of IT2 FSs and IT2 TrFNs. Let $X$ be a crisp set. A mapping $\tilde{A}: X \rightarrow[0,1]^{[0,1]}$ is called a T2 FS defined on the universe of discourse $X$. The T2 FS $\tilde{A}$ is expressed as follows (Mendel, 2001; Moharrer et al., 2015):

$$
\begin{aligned}
\tilde{A}= & \left\{( x , \mu _ { \tilde { A } } ( x ) ) \left\{\mid x \in X, \mu_{\tilde{A}}(x)=\right.\right. \\
& \left.\left\{\left(u, f_{x}(u)\right) \mid u \in J_{x} \subseteq[0,1], f_{x}(u) \in[0,1]\right\}\right\},
\end{aligned}
$$

where $\mathrm{x}$ is called a primary variable, $\mu_{\tilde{A}}(x)$ denotes the fuzzy membership value of $\mathrm{x}$ in $\tilde{A}$, $\mathrm{u}$ indicates the primary membership (grade) of $\mathrm{x}$, and $f_{x}(u)$ denotes the secondary membership (grade). Note that $\mu_{\tilde{A}}(x)$ is also known as a secondary membership function or a secondary set. Moreover, $J_{x} \subseteq[0,1]$ denotes the domain of $f_{x}(u)$ and represents the primary membership values of $x \in X$.

Let $\tilde{A}$ be a T2 FS on X. When $f_{x}(u)=1$ for all $u \in J_{x}, \tilde{A}$ is known as an IT2 FS on X and can be represented by (Mendel, 2001; Moharrer et al., 2015):

$$
\begin{aligned}
\tilde{A}= & \left\{\left(x, \mu_{\tilde{A}}(x)\right) \mid x \in X, \mu_{\tilde{A}}(x)=\right. \\
& \left\{(u, 1) \mid \mu_{\tilde{A}^{L}}(x) \leq u \leq \mu_{\tilde{A}^{U}}(x),(2)\right. \\
& {\left.\left.\left[\mu_{\tilde{A}^{L}}(x), \mu_{\tilde{A}^{U}}(x)\right] \subseteq[0,1]\right\}\right\}, }
\end{aligned}
$$

where $\mu_{\tilde{A}}(x)$ is referred to an interval membership value bounded within $\left[\mu_{\tilde{A}^{L}}(x), \mu_{\tilde{A}^{U}}(x)\right]$.

Let $\tilde{A}$ be an IT2 FS on X. $\tilde{A}$ can be fully characterized by its footprint of uncertainty (FOU), which is defined as the union of all primary memberships as follows (Mendel, 2001; Mendel and John, 2002; Moharrer et al., 2015; Singh and Garg, 2017):

$$
\operatorname{FOU}(\tilde{A})=\cup_{x \in X}\left[\mu_{\tilde{A}^{L}}(x), \mu_{\tilde{A}^{U}}(x)\right] .(3)
$$

$\operatorname{FOU}(\tilde{A})$ is a bounded region which represents the uncertainty associated with the membership grades of $\tilde{A}$.

A lower membership function (LMF) and an upper membership function (UMF) are two ordinary membership functions that are bounds for the $F O U(\tilde{A})$ of an IT2 FS $\tilde{A}$. Let two T1 FSs $\tilde{A}^{L}: \mathrm{X} \rightarrow[0,1]$ and $\tilde{A}^{U}: \mathrm{X} \rightarrow[0,1]$ be the lower and upper fuzzy sets, respectively, with respect to $\tilde{A}$. The LMF $\mu_{\tilde{A}^{L}}(x)$ and the UMF $\mu_{\tilde{A}^{U}}(x)$ are associated with the lower bound $\operatorname{FOU}^{L}(\tilde{A})$ and the upper bound $\operatorname{FOU}^{U}(\tilde{A})$, respectively, of $F O U(\tilde{A})$. They are defined as follows (Mendel, 2001; Zhao and Xiao, 2014; Zhao et al., 2014): 


$$
\begin{aligned}
& \tilde{A}^{L}=\operatorname{FOU}^{L}(\tilde{A})=\left\{\left(x, \mu_{\tilde{A}^{L}}(x)\right) \mid x \in X\right\},(4) \\
& \tilde{A}^{U}=\operatorname{FOU}^{U}(\tilde{A})=\left\{\left(x, \mu_{\tilde{A}^{U}}(x)\right) \mid x \in X\right\},(5)
\end{aligned}
$$

where $0 \leq \mu_{\tilde{A}^{L}}(x) \leq \mu_{\tilde{A}^{U}}(x) \leq 1$ for all $x \in X$.

Let $\tilde{A}$ be an IT2 FS on $X$. Let $\tilde{A}^{L}\left(=\left(a_{1}^{L}, a_{2}^{L}, a_{3}^{L}, a_{4}^{L} ; h_{\tilde{A}}^{L}\right)\right)$ and $\tilde{A}^{U}\left(=\left(a_{1}^{U}, a_{2}^{U}, a_{3}^{U}, a_{4}^{U} ; h_{\tilde{A}}^{U}\right)\right)$ be the lower and upper trapezoidal fuzzy numbers, respectively, with respect to $\tilde{A}$, where $\quad a_{1}^{L} \leq a_{2}^{L} \leq a_{3}^{L} \leq a_{4}^{L} \quad, \quad a_{1}^{U} \leq a_{2}^{U} \leq a_{3}^{U} \leq a_{4}^{U}$, $0 \leq h_{\tilde{A}}^{L} \leq h_{\tilde{A}}^{U} \leq 1, a_{1}^{U} \leq a_{1}^{L}, a_{4}^{L} \leq a_{4}^{U}$, and $\tilde{A}^{L} \subseteq \tilde{A}^{U}$ (i.e., if and only if $\left.\forall x \in X, \mu_{\tilde{A}^{L}}(x) \leq \mu_{\tilde{A}^{U}}(x)\right)$. The membership functions of $x$ in $\tilde{A}^{L}$ and $\tilde{A}^{U}$ are expressed as follows (Chen, 2013; 2017; Wang and Chen, 2015):

$$
\begin{gathered}
\mu_{\tilde{A}^{L}}(x)= \begin{cases}h_{\tilde{A}}^{L}\left(x-a_{1}^{L}\right) /\left(a_{2}^{L}-a_{1}^{L}\right) & \text { for } a_{1}^{L} \leq x \leq a_{2}^{L}, \\
h_{\tilde{A}}^{L} & \text { for } a_{2}^{L} \leq x \leq a_{3}^{L}, \\
h_{\tilde{A}}^{L}\left(a_{4}^{L}-x\right) /\left(a_{4}^{L}-a_{3}^{L}\right) & \text { for } a_{3}^{L} \leq x \leq a_{4}^{L}, \\
0 & \text { otherwise; }\end{cases} \\
\mu_{\tilde{A}^{U}}(x)= \begin{cases}h_{\tilde{A}}^{U}\left(x-a_{1}^{U}\right) /\left(a_{2}^{U}-a_{1}^{U}\right) & \text { for } a_{1}^{U} \leq x \leq a_{2}^{U}, \\
h_{\tilde{A}}^{U} & \text { for } a_{2}^{U} \leq x \leq a_{3}^{U}, \\
h_{\tilde{A}}^{U}\left(a_{4}^{U}-x\right) /\left(a_{4}^{U}-a_{3}^{U}\right) & \text { for } a_{3}^{U} \leq x \leq a_{4}^{U}, \\
0 & \text { otherwise. }\end{cases}
\end{gathered}
$$

The LMF $\mu_{\tilde{A}^{L}}(x)$ and the UMF $\mu_{\tilde{A}^{U}}(x)$ are lower and upper bounds, respectively, for the $\operatorname{FOU}(\tilde{A})$ of $\tilde{A}$. Then, $\tilde{A}$ is an IT2 TrFN on $X$ (see Figure 1 for a geometrical interpretation) and can be represented by:

$$
\tilde{A}=\left[\tilde{A}^{L}, \tilde{A}^{U}\right]=\left[\left(a_{1}^{L}, a_{2}^{L}, a_{3}^{L}, a_{4}^{L} ; h_{\tilde{A}}^{L}\right),\left(a_{1}^{U}, a_{2}^{U}, a_{3}^{U}, a_{4}^{U} ; h_{\tilde{A}}^{U}\right)\right] .
$$

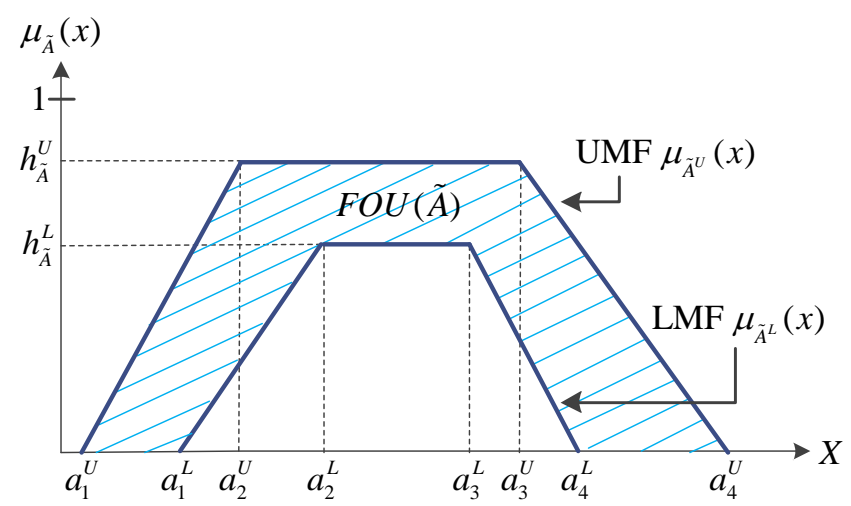

Fig 1: A geometrical interpretation of an IT2 TrFN $\tilde{A}$.

\section{MCDA USING A LIKELIHOOD-BASED COMPROMISE APPROACH}

This section develops a novel MCDA method using a likelihood-based compromise approachin the decision context of IT2 TrFNs. Several new concepts (e.g., likelihood indices, weighted likelihood indices, likelihood-based compromise indices, and likelihood-based compromise values) are developed to address the MCDA problems based on IT2 TrFNs and to avoid the computational complexity attached to IT2 FSs.

\subsection{Decision Environment under Study}

This subsection formulates an MCDA problem based on IT2 TrFNs within the decision environment of IT2 FSs. Consider an MCDA problem in which both the evaluative ratings of alternatives and the importance weights of criteria are expressed as IT2 TrFNs. Define $Z=\left\{z_{1}, z_{2}, \cdots, z_{m}\right\}$ as the set of decision alternatives, where $m$ is the number of alternatives. Define $C=\left\{c_{1}, c_{2}, \cdots, c_{n}\right\}$ as the set of evaluative criteria, where $n$ is the number of criteria.Let $x_{j}$ denote the value that corresponds to the criterion $c_{j}$, where $x_{j} \in X$ (i.e., the universe of discourse) for $j=1,2, \cdots, n$. The set $C$ can generally be divided into two sets, $C_{\mathrm{I}}$ and $C_{\mathrm{II}}$, where $C_{\mathrm{I}}$ denotes a collection of benefit criteria (i.e., a larger value of $x_{j}$ indicates a greater preference), $C_{\mathrm{II}}$ denotes a collection of cost criteria (i.e., a smaller value of $x_{j}$ indicates a greater preference), and where $C_{\mathrm{I}} \cap C_{\mathrm{II}}=\varnothing$ and $C_{\mathrm{I}} \cup C_{\mathrm{II}}=C$.

Let an IT2 $\operatorname{TrFN} \tilde{A}_{i j}$ denote the evaluative rating of an alternative $z_{i} \in Z$ with respect to criterion $c_{j} \in C$; it is expressed as follows:

$$
\begin{aligned}
\tilde{A}_{i j} & =\left[\tilde{A}_{i j}^{L}, \tilde{A}_{i j}^{U}\right] \\
& =\left[\left(a_{1 i j}^{L}, a_{2 i j}^{L}, a_{3 i j}^{L}, a_{4 i j}^{L} ; h_{\tilde{A}_{i j}}^{L}\right),\left(a_{1 i j}^{U}, a_{2 i j}^{U}, a_{3 i j}^{U}, a_{4 i j}^{U} ; h_{\tilde{A}_{i j}}^{U}\right)\right],
\end{aligned}
$$

where $\quad \tilde{A}_{i j}^{L} \quad\left(=\left(a_{1 i j}^{L}, a_{2 i j}^{L}, a_{3 i j}^{L}, a_{4 i j}^{L} ; h_{\tilde{A}_{i j}}^{L}\right) \quad\right) \quad$ and $\quad \tilde{A}_{i j}^{U} \quad($ $\left.=\left(a_{1 i j}^{U}, a_{2 i j}^{U}, a_{3 i j}^{U}, a_{4 i j}^{U} ; h_{\tilde{A}_{i j}}^{U}\right)\right)$ denote the lower and upper trapezoidal fuzzy numbers, respectively, with respect to $\tilde{A}_{i j}$. The characteristics of the alternative $z_{i}$ can be represented by:

$$
\begin{aligned}
\tilde{A}_{i}= & \left\{\left\langle c_{j},\left[\tilde{A}_{i j}^{L}, \tilde{A}_{i j}^{U}\right]\right\rangle \mid c_{j} \in C\right\} \\
= & \left\{\left\langlec_{j},\left[\left(a_{1 i j}^{L}, a_{2 i j}^{L}, a_{3 i j}^{L}, a_{4 i j}^{L} ; h_{\tilde{A}_{i j}}^{L}\right),\right.\right.\right. \\
& \left.\left.\left.\left(a_{1 i j}^{U}, a_{2 i j}^{U}, a_{3 i j}^{U}, a_{4 i j}^{U} ; h_{\tilde{A}_{i j}}^{U}\right)\right]\right\rangle c_{j} \in C\right\} .
\end{aligned}
$$


Let an IT2 $\operatorname{TrFN} \tilde{W}_{j}$ denote the importance weight of a criterion $c_{j} \in C$; it is expressed as follows:

$$
\begin{aligned}
\tilde{W}_{j} & =\left[\tilde{W}_{j}^{L}, \tilde{W}_{j}^{U}\right] \\
& =\left[\left(w_{1 j}^{L}, w_{2 j}^{L}, w_{3 j}^{L}, w_{4 j}^{L} ; h_{\tilde{W}_{j}}^{L}\right),\left(w_{1 j}^{U}, w_{2 j}^{U}, w_{3 j}^{U}, w_{4 j}^{U} ; h_{\tilde{W}_{j}}^{U}\right)\right],
\end{aligned}
$$

where $\tilde{W}_{j}^{L} \quad\left(=\left(w_{1 j}^{L}, w_{2 j}^{L}, w_{3 j}^{L}, w_{4 j}^{L} ; h_{\tilde{W}_{j}}^{L}\right)\right)$ and $\tilde{W}_{j}^{U}($ $\left.=\left(w_{1 j}^{U}, w_{2 j}^{U}, w_{3 j}^{U}, w_{4 j}^{U} ; h_{\tilde{w}_{j}}^{U}\right)\right)$ denote the lower and upper trapezoidal fuzzy numbers, respectively, with respect to $\tilde{W}_{j}$. The collection of the importance weights for all $c_{j} \in C$ can be represented by:

$$
\begin{aligned}
\tilde{W}= & \left\{\left\langle c_{j},\left[\tilde{W}_{j}^{L}, \tilde{W}_{j}^{U}\right]\right\rangle \mid c_{j} \in C\right\} \\
= & \left\{\left\langlec_{j},\left[\left(w_{1 j}^{L}, w_{2 j}^{L}, w_{3 j}^{L}, w_{4 j}^{L} ; h_{\tilde{W}_{j}}^{L}\right),\right.\right.\right. \\
& \left.\left.\left.\left(w_{1 j}^{U}, w_{2 j}^{U}, w_{3 j}^{U}, w_{4 j}^{U} ; h_{\tilde{W}_{j}}^{U}\right)\right]\right\rangle \mid c_{j} \in C\right\} .
\end{aligned}
$$

Note that it is reasonable for the most part to assume $w_{\eta j}^{L}>0, w_{\eta j}^{U}>0$, and $h_{\tilde{W}_{j}}^{U}>0$ for $\eta \in\{1,2,3,4\}$ and $c_{j} \in C$ because all of the criteria in $C$ are salient attributes that provide a means of alternative evaluations.

\subsection{Proposed Method}

This subsection presents a simple and useful MCDA method using a likelihood-based compromise approach in the decision context of IT2 TrFNs. First, the concept of approximate positive-ideal and negative-ideal solutions is introduced to facilitate anchored judgments concerning the evaluative ratings of alternatives. Next, based on the likelihood of an IT2 TrFN binary relation, this subsection establishes useful concepts of likelihood indices and weighted likelihood indices relative to the approximate ideals. Finally, this subsection proposes a novel likelihoodbased compromise index of the characteristics for each alternative and then determines the corresponding likelihood-based compromise value using a signed distancebased method for acquiring the priority orders of the alternatives.

MCDA problems address the ranking of alternatives and the selection of the best compromise alternative among a finite set of alternatives based on a finite set of criteria. Considering the issue of anchor dependency, the best compromise alternative is the solution that should be as close as possible to the positive-ideal solution and as far away from the negative-ideal solution as possible concurrently. In a similar manner to the technique for order preference by similarity to ideal solution (TOPSIS), the positive-ideal and negative-ideal solutions should be directly generated from the characteristics of all of the alternatives for the purpose of the points of reference in the decisionmaking process. Specifically, the positive-ideal solution is composed of all best criterion values attainable, while the negative-ideal solution is composed of all worst criterion values attainable. However, applying Zadeh's extension principle (Zadeh, 1975), the evaluative ratings of the positive-ideal and negative-ideal solutions are not IT2 TrFNs in general. Because the computational manipulation with IT2 TrFNs is relatively easy and simple, this paper employs the concept of approximate positive-ideal and negative-ideal solutions to simplify mathematical calculations and enhance the implementation efficiency in the proposed MCDA method.

Let $z_{*}$ and $z_{-}$denote the approximate positive-ideal and negative-ideal solutions, respectively; moreover, theirevaluative ratings are expressed as IT2 TrFNs. Let an IT2 $\operatorname{TrFN} \tilde{A}_{* j}$ denote the evaluative rating of the approximate positive-ideal solution $z_{*}$ with respect to criterion $c_{j} \in C$; it is represented by:

$$
\begin{aligned}
\tilde{A}_{* j}= & {\left[\tilde{A}_{*_{j}}^{L}, \tilde{A}_{* j}^{U}\right] } \\
= & {\left[\left(a_{1^{* j}}^{L}, a_{2^{* j}}^{L}, a_{3^{* j}}^{L}, a_{4^{* j}}^{L} ; h_{\tilde{A}_{*_{j}}}^{L}\right),\right.} \\
& \left.\left(a_{1^{* j} j}^{U}, a_{2^{* j} j}^{U}, a_{3^{* j} j}^{U}, a_{4 * j}^{U} ; h_{\tilde{A}_{* j}}^{U}\right)\right],
\end{aligned}
$$

where $\tilde{A}_{* j}^{L} \quad\left(=\left(a_{1^{* j} j}^{L}, a_{2^{* j} j}^{L}, a_{3^{* j} j}^{L}, a_{4 * j}^{L} ; h_{\tilde{A}_{* j}}^{L}\right)\right)$ and $\tilde{A}_{*_{j}}^{U} \quad($ $\left.=\left(a_{1 * j}^{U}, a_{2^{* j}}^{U}, a_{3^{* j}}^{U}, a_{4 *_{j}}^{U} ; h_{\tilde{A}_{* j}}^{U}\right)\right)$ denote the lower and upper trapezoidal fuzzy numbers, respectively, with respect to $\tilde{A}_{{ }_{j}}$ . In this work, $\tilde{A}_{* j}^{L}$ and $\tilde{A}_{* j}^{U}$ are defined as follows:

$$
\begin{gathered}
\tilde{A}_{* j}^{L}=\left\{\begin{array}{c}
\left(\max _{i=1}^{m} a_{1 i j}^{L}, \max _{i=1}^{m} a_{2 i j}^{L}, \max _{i=1}^{m} a_{3 i j}^{L}, \max _{i=1}^{m} a_{4 i j}^{L} ; \min _{i=1}^{m} h_{\tilde{A}_{i j}}^{L}\right.
\end{array}\right) \\
\text { if } c_{j} \in C_{\mathrm{I}}, \\
\left(\begin{array}{c}
m \\
\min _{i=1}^{m} a_{1 i j}^{L}, \min _{i=1}^{m} a_{2 i j}^{L}, \min _{i=1}^{m} a_{3 i j}^{L}, \min _{i=1}^{m} a_{4 i j}^{L} ; \min _{i=1}^{m} h_{\tilde{A}_{i j}}^{L}
\end{array}\right) \\
\text { if } c_{j} \in C_{\mathrm{II}} ; \\
\tilde{A}_{* j}^{U}=\left\{\begin{array}{c}
\max _{i=1}^{m} a_{1 i j}^{U}, \max _{i=1}^{m} a_{2 i j}^{U}, \max _{i=1}^{m} a_{3 i j}^{U}, \max _{i=1}^{m} a_{4 i j}^{U} ; \min _{i=1}^{m} h_{\tilde{A}_{i j}}^{U}
\end{array}\right) \\
\left(\begin{array}{c}
m \\
\min _{i=1}^{m} a_{1 i j}^{U}, \min _{i=1}^{m} a_{2 i j}^{U}, \min _{i=1}^{m} a_{3 i j}^{U}, \min _{i=1}^{m} a_{4 i j}^{U} ; \min _{i=1}^{m} h_{\tilde{A}_{i j}}^{U}
\end{array}\right)
\end{gathered}
$$


Accordingly, the characteristics of $z_{*}$ is expressed as follows:

$$
\begin{aligned}
\tilde{A}_{*}= & \left\{\left\langle c_{j},\left[\tilde{A}_{* j}^{L}, \tilde{A}_{* j}^{U}\right]\right\rangle \mid c_{j} \in C\right\} \\
= & \left\{\left\langlec_{j},\left[\left(a_{1^{*} j}^{L}, a_{2^{*} j}^{L}, a_{3^{*} j}^{L}, a_{4^{* j}}^{L} ; h_{\tilde{A}_{* j}}^{L}\right),\right.\right.\right. \\
& \left.\left.\left.\left(a_{1^{*} j}^{U}, a_{2^{*} j}^{U}, a_{3^{*} j}^{U}, a_{4^{*} j}^{U} ; h_{\tilde{A}_{* j}}^{U}\right)\right]\right\rangle \mid c_{j} \in C\right\} .
\end{aligned}
$$

Similarly, let an IT2 $\operatorname{TrFN} \tilde{A}_{-j}$ denote the evaluative rating of the approximate negative-ideal solution $z_{-}$with respect to criterion $c_{j} \in C$, and it is represented by:

$$
\begin{aligned}
\tilde{A}_{-j}= & {\left[\tilde{A}_{-j}^{L}, \tilde{A}_{-j}^{U}\right] } \\
= & {\left[\left(a_{1-j}^{L}, a_{2-j}^{L}, a_{3-j}^{L}, a_{4-j}^{L} ; h_{\tilde{A}_{-j}}^{L}\right),\right.} \\
& \left.\left(a_{1-j}^{U}, a_{2-j}^{U}, a_{3-j}^{U}, a_{4-j}^{U} ; h_{\tilde{A}_{-j}}^{U}\right)\right],
\end{aligned}
$$

where $\tilde{A}_{-j}^{L} \quad\left(=\left(a_{1-j}^{L}, a_{2-j}^{L}, a_{3-j}^{L}, a_{4-j}^{L} ; h_{\tilde{A}_{-j}}^{L}\right)\right)$ and $\tilde{A}_{-j}^{U}$ $\left.=\left(a_{1-j}^{U}, a_{2-j}^{U}, a_{3-j}^{U}, a_{4-j}^{U} ; h_{\tilde{A}_{-j}}^{U}\right)\right)$ denote the lower and upper trapezoidal fuzzy numbers, respectively, with respect to $\tilde{A}_{-j}$. Specifically, they are defined as follows:

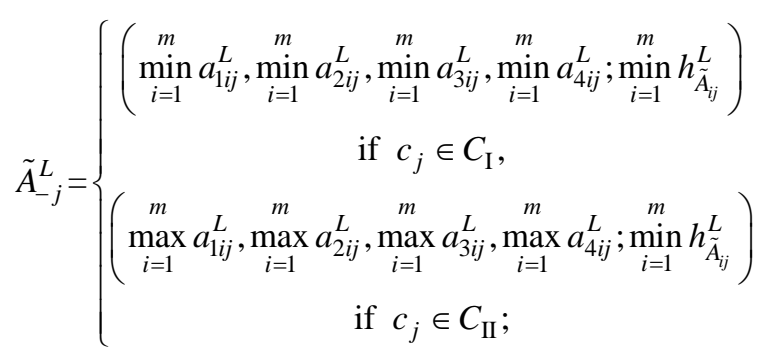

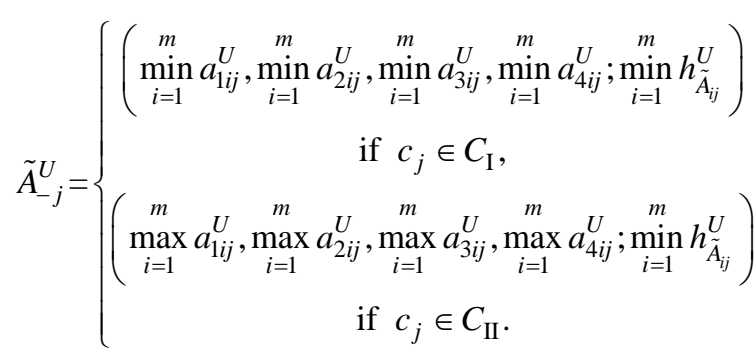

Next, the characteristics of $z_{-}$is expressed as follows:

$$
\begin{aligned}
\tilde{A}_{-}= & \left\{\left\langle c_{j},\left[\tilde{A}_{-j}^{L}, \tilde{A}_{-j}^{U}\right]\right\rangle \mid c_{j} \in C\right\} \\
= & \left\{\left\langlec_{j},\left[\left(a_{1-j}^{L}, a_{2-j}^{L}, a_{3-j}^{L}, a_{4-j}^{L} ; h_{\tilde{A}_{-j}}^{L}\right),\right.\right.\right. \\
& \left.\left.\left.\left(a_{1-j}^{U}, a_{2-j}^{U}, a_{3-j}^{U}, a_{4-j}^{U} ; h_{\tilde{A}_{-j}}^{U}\right)\right]\right\rangle \mid c_{j} \in C\right\} .
\end{aligned}
$$

In regard to the benefit criterion $c_{j} \in C_{\mathrm{I}}$, the lower and upper fuzzy sets with respect to $\tilde{A}_{*_{j}}$ can serve as the upper bounds of those with respect to the associated evaluative rating of the positive-ideal solution, while the lower and upper fuzzy sets with respect to $\tilde{A}_{-j}$ can serve as the lower bounds of those with respect to the associated evaluative rating of the negative-ideal solution. In contrast, for the cost criterion $c_{j} \in C_{\mathrm{II}}$, the lower and upper fuzzy sets with respect to $\tilde{A}_{{ }_{j}}$ can serve as lower bounds of those with respect to the associated evaluative rating of the positiveideal solution, while the lower and upper fuzzy sets with respect to $\tilde{A}_{-j}$ can serve as upper bounds of those with respect to the associated evaluative rating of the negativeideal solution. In this regard, it is appropriate to approximate the positive-ideal and negative-ideal solutions by $z_{*}$ and $z_{-}$, respectively.

Based on the likelihood of an IT2 TrFN binary relation, this paper attempts to develop a novel likelihood-based compromise approach for the purpose of evaluating alternatives. An approach using likelihood-based comparisons has been applied to address MCDA problems (Chen and Lee, 2010a; 2010b; 2010c; Li, 2010a; 2010b; 2011; Wang et al., 2015). This paper uses the extended concept of likelihoods proposed by Wang et al. (2015) to compare the IT2 TrFN evaluative ratings.

Consider any two IT2 TrFN evaluative ratings $\tilde{A}_{i j}$ and $\tilde{A}_{i^{\prime} j}$ of the alternatives $z_{i}$ and $z_{i^{\prime}}$, respectively, with respect to criterion $c_{j} \in C$. Assume that at least one of $h_{\tilde{A}_{i j}}^{-} \neq h_{\tilde{A}_{i^{\prime} j}}^{+}$, $a_{4 i j}^{-} \neq a_{1 i j}^{-}, a_{4 i^{\prime} j}^{+} \neq a_{1^{\prime} j}^{+}$, and $a_{\eta i j}^{-} \neq a_{\eta i^{\prime} j}^{+}$holds, and at least one of $h_{\tilde{A}_{i j}}^{+} \neq h_{\tilde{A}_{i^{\prime} j}}, a_{4 i j}^{+} \neq a_{1 i j}^{+}, a_{4 i^{\prime} j}^{-} \neq a_{1 i^{\prime} j}^{-}$, and $a_{\eta i j}^{+} \neq a_{\eta i^{\prime} j}^{-}$ holds, where $\eta \in\{1,2,3,4\}$. The lower likelihood $L^{L}\left(\tilde{A}_{i j} \geq \tilde{A}_{i^{\prime} j}\right)$ and the upper likelihood $L^{U}\left(\tilde{A}_{i j} \geq \tilde{A}_{i^{\prime} j}\right)$ of an IT2 TrFN binary relation $\tilde{A}_{i j} \geq \tilde{A}_{i^{\prime} j}$ are defined as follows:

$$
\begin{aligned}
L^{L}\left(\tilde{A}_{i j} \geq \tilde{A}_{i^{\prime} j}\right)= & \max \left\{1-\max \left[\sum_{\eta=1}^{4} \max \left(a_{\eta i^{\prime} j}^{+}-a_{\eta i^{j}}^{-}, 0\right)+\right.\right. \\
& \left(a_{4 i^{\prime} j}^{+}-a_{1 i j}^{-}\right)+2 \max \left(h_{\tilde{A}_{i^{\prime} j}^{+}}^{+}-h_{\tilde{A}_{i j}}^{-}, 0\right) / \sum_{\eta=1}^{4}\left|a_{\eta i^{\prime} j}^{+}-a_{\eta i j}^{-}\right|+ \\
& \left.\left.\left(a_{4 i j}^{-}-a_{1 i j}^{-}\right)+\left(a_{4 i^{\prime} j}^{+}-a_{i^{\prime} j}^{+}\right)+2\left|h_{\tilde{A}_{i^{\prime} j}^{+}}^{+}-h_{\tilde{A}_{i j}}^{-}\right|, 0\right], 0\right\},
\end{aligned}
$$




$$
\begin{aligned}
L^{U}\left(\tilde{A}_{i j} \geq \tilde{A}_{i^{\prime} j}\right)= & \max \left\{1-\max \left[\sum_{\eta=1}^{4} \max \left(a_{\eta i^{\prime} j}^{-}-a_{\eta i j}^{+}, 0\right)+\right.\right. \\
& \left(a_{4 i^{\prime} j}^{-}-a_{1 i j}^{+}\right)+2 \max \left(h_{\tilde{A}_{i^{\prime} j}}^{-}-h_{\tilde{A}_{i j}}^{+}, 0\right) / \sum_{\eta=1}^{4}\left|a_{\eta i^{\prime} j}^{-}-a_{\eta i j}^{+}\right|+ \\
& \left.\left.\left(a_{4 i j}^{+}-a_{1 i j}^{+}\right)+\left(a_{4 i^{\prime} j}^{-}-a_{1 i^{\prime} j}^{-}\right)+2\left|h_{\tilde{A}_{i^{\prime} j}^{-}}^{-}-h_{\tilde{A}_{i j}}^{+}\right|, 0\right], 0\right\} .
\end{aligned}
$$

The likelihood $L\left(\tilde{A}_{i j} \geq \tilde{A}_{i^{\prime} j}\right)$ of the IT2 TrFN binary relation $\tilde{A}_{i j} \geq \tilde{A}_{i^{\prime} j}$ is defined as follows:

$$
L\left(\tilde{A}_{i j} \geq \tilde{A}_{i^{\prime} j}\right)=\frac{L^{L}\left(\tilde{A}_{i j} \geq \tilde{A}_{i^{\prime} j}\right)+L^{U}\left(\tilde{A}_{i j} \geq \tilde{A}_{i^{\prime} j}\right)}{2},
$$

where it satisfies the following useful properties: (i) $0 \leq L\left(\tilde{A}_{i j} \geq \tilde{A}_{i^{\prime} j}\right) \leq 1 \quad$; (ii) $L\left(\tilde{A}_{i j} \geq \tilde{A}_{i^{\prime} j}\right)+L\left(\tilde{A}_{i^{\prime} j} \geq \tilde{A}_{i j}\right)=1$; (iii) $\quad L\left(\tilde{A}_{i j} \geq \tilde{A}_{i^{\prime} j}\right)=L\left(\tilde{A}_{i^{\prime} j} \geq \tilde{A}_{i j}\right)=0.5 \quad$ if $\quad L\left(\tilde{A}_{i j} \geq \tilde{A}_{i^{\prime} j}\right)$ $=L\left(\tilde{A}_{i^{\prime} j} \geq \tilde{A}_{i j}\right) \quad ; \quad$ (iv) $\quad L\left(\tilde{A}_{i j} \geq \tilde{A}_{i j}\right)=0.5 \quad ; \quad$ and $\quad$ (v) $\sum_{i=1}^{m} \sum_{i^{\prime}=1}^{m} L\left(\tilde{A}_{i j} \geq \tilde{A}_{i^{\prime} j}\right)=0.5 \cdot m^{2}$.

This paper makes an effective use of the likelihoods of IT2 TrFN binary relations to compare the IT2 TrFN evaluative ratings of a specific alternative and the approximate ideal solutions. Let $L I_{i j}^{*}$ denote the likelihood index of $\tilde{A}_{i j}$ relative to $\tilde{A}_{* j}$ for each $z_{i} \in Z$ and $c_{j} \in C . L I_{i j}^{*}$ is defined as the likelihood of the IT2 TrFN binary relation of $\tilde{A}_{i j}$ not being larger than $\tilde{A}_{*_{j}}$ (i.e., $\tilde{A}_{i j} \leq \tilde{A}_{*_{j}}$ ) for $c_{j} \in C_{\mathrm{I}}$ and the likelihood of the IT2 TrFN binary relation of $\tilde{A}_{i j}$ not being smaller than $\tilde{A}_{* j}$ (i.e., $\tilde{A}_{i j} \geq \tilde{A}_{*_{j}}$ ) for $c_{j} \in C_{\mathrm{II}}$. That is,

$$
L I_{i j}^{*}= \begin{cases}L\left(\tilde{A}_{* j} \geq \tilde{A}_{i j}\right) & \text { if } c_{j} \in C_{\mathrm{I}}, \\ L\left(\tilde{A}_{i j} \geq \tilde{A}_{* j}\right) & \text { if } c_{j} \in C_{\mathrm{II}},\end{cases}
$$

where $0<L I_{i j}^{*} \leq 1$. In contrast, let $L I_{i j}^{-}$denote the likelihood index of $\tilde{A}_{i j}$ relative to $\tilde{A}_{-j}$ for each $z_{i} \in Z$ and $c_{j} \in C$. Specifically, $L I_{i j}^{-}$is determined via the likelihood of the IT2 TrFN binary relation of $\tilde{A}_{i j}$ not being smaller than $\tilde{A}_{-j}$ (i.e., $\tilde{A}_{i j} \geq \tilde{A}_{-j}$ ) for $c_{j} \in C_{\mathrm{I}}$ and the likelihood of the IT2 TrFN binary relation of $\tilde{A}_{i j}$ not being larger than $\tilde{A}_{-j}$ (i.e., $\tilde{A}_{i j} \leq \tilde{A}_{-j}$ ) for $c_{j} \in C_{\mathrm{II}}$. That is,

$$
L I_{i j}^{-}= \begin{cases}L\left(\tilde{A}_{i j} \geq \tilde{A}_{-j}\right) & \text { if } c_{j} \in C_{\mathrm{I}}, \\ L\left(\tilde{A}_{-j} \geq \tilde{A}_{i j}\right) & \text { if } c_{j} \in C_{\mathrm{II}}\end{cases}
$$

where $0<L I_{i j}^{-} \leq 1$.

Next, the concept of weighted likelihood indices can be established via combining the likelihood indices with the IT2 TrFN importance weight $\tilde{W}_{j}$ for all $c_{j} \in C$. Let two IT2 TrFNs $L \tilde{I}_{i j}^{*}$ and $L \tilde{I}_{i j}^{-}$denote the weighted likelihood indices of $\tilde{A}_{i j}$ relative to $\tilde{A}_{*_{j}}$ and $\tilde{A}_{-j}$, respectively; they are expressed as follows:

$$
\begin{aligned}
L \tilde{I}_{i j}^{*}= & {\left[L \tilde{I}_{i j}^{* L}, L \tilde{I}_{i j}^{* U}\right] } \\
= & {\left[\left(l i_{1 i j}^{* L}, l_{2 i j}^{* L}, l i_{3 i j}^{* L}, l i_{4 i j}^{* L} ; h_{L \tilde{I}_{i j}^{*}}^{L}\right),\right.} \\
& \left.\left(l i_{1 i j}^{* U}, l i_{2 i j}^{* U}, l i_{3 i j}^{* U}, l i_{4 i j}^{* U} ; h_{L \tilde{I}_{i j}^{*}}^{U}\right)\right], \\
L \tilde{I}_{i j}^{-}= & {\left[L \tilde{I}_{i j}^{-L}, L \tilde{I}_{i j}^{-U}\right] } \\
= & {\left[\left(l i_{1 i j}^{-L}, l i_{2 i j}^{-L}, l i_{3 i j}^{-L}, l i_{4 i j}^{-L} ; h_{L \tilde{I}_{i j}^{-}}^{L}\right),\right.} \\
& \left.\left(l i_{1 i j}^{-U}, l i_{2 i j}^{-U}, l i_{3 i j}^{-U}, l i_{4 i j}^{-U} ; h_{L \tilde{I}_{i j}^{-}}^{U}\right)\right] .
\end{aligned}
$$

Specifically, $L \tilde{I}_{i j}^{*}$ and $L \tilde{I}_{i j}^{-}$can be determined in the following manner:

$$
\begin{aligned}
L \tilde{I}_{i j}^{*}= & {\left[\left(L I_{i j}^{*} \cdot w_{1 j}^{L}, L I_{i j}^{*} \cdot w_{2 j}^{L}, L I_{i j}^{*} \cdot w_{3 j}^{L}, L I_{i j}^{*} \cdot w_{4 j}^{L} ; h_{\tilde{W}_{j}}^{L}\right),\right.} \\
& \left.\left(L I_{i j}^{*} \cdot w_{1 j}^{U}, L I_{i j}^{*} \cdot w_{2 j}^{U}, L I_{i j}^{*} \cdot w_{3 j}^{U}, L I_{i j}^{*} \cdot w_{4 j}^{U} ; h_{\tilde{W}_{j}}^{U}\right)\right], \\
L \tilde{I}_{i j}^{-}= & {\left[\left(L I_{i j}^{-} \cdot w_{1 j}^{L}, L I_{i j}^{-} \cdot w_{2 j}^{L}, L I_{i j}^{-} \cdot w_{3 j}^{L}, L I_{i j}^{-} \cdot w_{4 j}^{L} ; h_{\tilde{W}_{j}}^{L}\right),\right.} \\
& \left.\left(L I_{i j}^{-} \cdot w_{1 j}^{U}, L I_{i j}^{-} \cdot w_{2 j}^{U}, L I_{i j}^{-} \cdot w_{3 j}^{U}, L I_{i j}^{-} \cdot w_{4 j}^{U} ; h_{\tilde{W}_{j}}^{U}\right)\right],
\end{aligned}
$$

for $i=1,2, \cdots, m$ and $j=1,2, \cdots, n$. Note that the likelihood indices $L I_{i j}^{*}$ and $L I_{i j}^{-}$are ordinary numbers, while the weighted likelihood indices $L \tilde{I}_{i j}^{*}$ and $L \tilde{I}_{i j}^{-}$are IT2 TrFNs. Additionally, for $\eta \in\{1,2,3,4\}$ and $c_{j} \in C$, the inequalities of $l i_{1 \eta j}^{* L}>0, l i_{1 \eta j}^{* U}>0, l i_{1 \eta j}^{-L}>0, l i_{1 \eta j}^{-U}>0, h_{L \tilde{I}_{i j}^{*}}^{U}>0$, and $h_{L \tilde{I}_{i j}^{-}}^{U}>0$ hold because of the conditions of $L I_{i j}^{*}>0$, $L I_{i j}^{-}>0, w_{\eta j}^{L}>0, w_{\eta j}^{U}>0$, and $h_{\tilde{W}_{j}}^{U}>0$.

In general, the alternative $z_{i} \in Z$ results in a good performance in terms of a benefit criterion $c_{j} \in C_{\mathrm{I}}$ when the IT2 $\operatorname{TrFN}$ evaluative rating $\tilde{A}_{i j}$ has a low possibility of being inferior to $\tilde{A}_{* j}$ and a high possibility of being superior to 
$\tilde{A}_{-j}$. In contrast, the alternative $z_{i} \in Z$ performs well with respect to a cost criterion $c_{j} \in C_{\mathrm{II}}$ when the IT2 $\operatorname{TrFN}$ evaluative rating $\tilde{A}_{i j}$ has a low possibility of being farther from $\tilde{A}_{*_{j}}$ and a high possibility of being farther from $\tilde{A}_{-j}$. Thus, it is anticipated that the lower the weighted likelihood index $L \tilde{I}_{i j}^{*}$ and the higher the $L \tilde{I}_{i j}^{-}$over all $n$ criteria, the better the alternative will be. However, as the whole, any alternative which has the lowest $L \tilde{I}_{i j}^{*}$ is not guaranteed to have the highest $L \tilde{I}_{i j}^{-}$in terms of a specific criterion $c_{j}$. Following the discussion described, this paper presents the likelihood-based compromise index $C \tilde{I}_{i}$ of the characteristics $\tilde{A}_{i}$ for the alternative $z_{i}$ as shown below:

$$
\begin{aligned}
C \tilde{I}_{i}= & {\left[C \tilde{I}_{i}^{L}, C \tilde{I}_{i}^{U}\right] } \\
= & {\left[\left(c i_{1 i}^{L}, c i_{2 i}^{L}, c i_{3 i}^{L}, c i_{4 i}^{L} ; h_{C \tilde{I}_{i}}^{L}\right),\right.} \\
& \left.\left(c i_{1 i}^{U}, c i_{2 i}^{U}, c i_{3 i}^{U}, c i_{4 i}^{U} ; h_{C \tilde{I}_{i}}^{U}\right)\right] .
\end{aligned}
$$

Let $\oplus$ and $\varnothing$ denote the operations of addition and division, respectively, on IT2 TrFNs (Chen, 2013; 2017; Chen et al., 2013). The likelihood-based compromise index $C \tilde{I}_{i}$ is determined in the following manner:

$$
C \tilde{I}_{i}=\left(\bigoplus_{j=1}^{n} L \tilde{I}_{i j}^{-}\right) \varnothing\left[\left(\bigoplus_{j=1}^{n} L \tilde{I}_{i j}^{*}\right) \oplus\left(\bigoplus_{j=1}^{n} L \tilde{I}_{i j}^{-}\right)\right],
$$

Where

$$
\begin{aligned}
& \bigoplus_{j=1}^{n} L \tilde{I}_{i j}^{*}=L \tilde{I}_{i 1}^{*} \oplus L \tilde{I}_{i 2}^{*} \oplus \cdots \oplus L \tilde{I}_{i n}^{*}, \\
& \bigoplus_{j=1}^{n} L \tilde{I}_{i j}^{-}=L \tilde{I}_{i 1}^{-} \oplus L \tilde{I}_{i 2}^{-} \oplus \cdots \oplus L \tilde{I}_{i n}^{-} .
\end{aligned}
$$

Applying the operations of addition and division on IT2 TrFNs, the lower and upper trapezoidal fuzzy numbers $C \tilde{I}_{i}^{L}$ and $C \tilde{I}_{i}^{U}$, respectively, with respect to $C \tilde{I}_{i}$ for $z_{i} \in Z$ are computed as follows:

$$
\begin{aligned}
C \tilde{I}_{i}^{L}= & \left(c i_{1 i}^{L}, c i_{2 i}^{L}, c i_{3 i}^{L}, c i_{4 i}^{L} ; h_{C \tilde{I}_{i}}^{L}\right) \\
= & \left(\sum_{j=1}^{n} l i_{1 i j}^{-L} / \sum_{j=1}^{n} l i_{4 i j}^{*}+\sum_{j=1}^{*} l i_{4 i j}^{-L}, \sum_{j=1}^{n} l i_{2 i j}^{-L} / \sum_{j=1}^{n} l i_{3 i j}^{*}+\sum_{j=1}^{n} l i_{3 i j}^{-L}\right. \\
& \sum_{j=1}^{n} l i_{3 i j}^{-L} / \sum_{j=1}^{n} l i_{2 i j}^{* L}+\sum_{j=1}^{n} l i_{2 i j}^{-L}, \sum_{j=1}^{n} l i_{4 i j}^{-L} / \sum_{j=1}^{n} l i_{1 i j}^{* L}+\sum_{j=1}^{n} l i_{1 i j}^{-L} \\
& \left.\min \left\{\min _{j=1}^{n} h_{L \tilde{I}_{i j}^{*}}^{L}, \min _{j=1}^{n} h_{L \tilde{I}_{i j}^{*}}^{L}\right\}\right) .
\end{aligned}
$$

$$
\begin{aligned}
C \tilde{I}_{i}^{U}= & \left(c i_{1 i}^{U}, c i_{2 i}^{U}, c i_{3 i}^{U}, c i_{4 i}^{U}, h_{C \tilde{I}_{i}}^{U}\right) \\
= & \left(\sum_{j=1}^{n} l i_{1 i j}^{-U} / \sum_{j=1}^{n} l i_{4 i j}^{*}+\sum_{j=1}^{n} l i_{4 i j}^{-U}, \sum_{j=1}^{n} l i_{2 i j}^{-U} / \sum_{j=1}^{n} l i_{3 i j}^{* U}+\sum_{j=1}^{n} l i_{3 i j}^{-U}\right. \\
& \sum_{j=1}^{n} l i_{3 i j}^{-U} / \sum_{j=1}^{n} l i_{2 i j}^{* U}+\sum_{j=1}^{n} l i_{2 i j}^{-U}, \sum_{j=1}^{n} l i_{4 i j}^{-U} / \sum_{j=1}^{n} l i_{1 i j}^{* U}+\sum_{j=1}^{n} l i_{l i j}^{-U} ; \\
& \left.\min \left\{\min _{j=1}^{n} h_{L \tilde{I}_{i j}^{*}}^{U}, \min _{j=1}^{n} h_{L \tilde{I}_{i j}^{*}}^{U}\right\}\right) .
\end{aligned}
$$

Note that the division operations in (34) and (35) are always meaningful because $\quad \sum_{j=1}^{n} l i_{\eta i j}^{* L}+\sum_{j=1}^{n} l i_{\eta i j}^{-L}>0 \quad$ and $\sum_{j=1}^{n} l i_{\eta i j}^{* U}+\sum_{j=1}^{n} l i_{\eta i j}^{-U}>0$ hold for $\eta \in\{1,2,3,4\}$.

The higher the likelihood-based compromise index $C \tilde{I}_{i}$, the better the alternative $z_{i}$ will be. Thus, this paper attempts to compare and rank the likelihood-based compromise indices of the $m$ alternatives to acquire the best compromise alternative. However, the likelihood-based compromise indices are IT2 TrFNs and cannot be compared easily. Accordingly, the signed distance-based method presented by Chen $(2013$; 2014a; 2017) can be used to obtain the comparable values of $C \tilde{I}_{i}$ for each $z_{i} \in Z$. Signed distances (i.e., an oriented distance or directed distance) have often been employed to determine the rankings of fuzzy numbers (Chen, 2013; 2017; Wang and Chen, 2015). Moreover, the concept of signed distances has been extended to the decision environment of IT2 TrFNs, and the signed distance-based method has been successfully employed to develop several useful MCDA methods (Chen, 2013; 2014a; 2014b; 2017; Chen et al., 2013; Wang and Chen, 2015). The signed distance-based method can use both positive and negative values to define the ordering ofIT2 TrFNs, which differs considerably from ordinary distance measures. In particular, signed distances provide a linear order that allows comparing IT2 TrFNs (Chen, 2014a).Therefore, the concept of signed distances is suggested for use in acquiring comparable values of the likelihood-based compromise index $C \tilde{I}_{i}$.

Let $C V_{i}$ denote the likelihood-based compromise value of the characteristics $\tilde{A}_{i}$ for the alternative $z_{i} \in Z$. In this work, $C V_{i}$ is determined using the signed distances from $C \tilde{I}_{i}$ to the level-1 fuzzy number mapping on the vertical axis at the origin of the coordinates, as shown below:

$$
\begin{aligned}
C V_{i}= & \frac{1}{8}\left[c i_{1 i}^{L}+c i_{2 i}^{L}+c i_{3 i}^{L}+c i_{4 i}^{L}+\frac{2 h_{C \tilde{I}_{i}}^{U}+3 h_{C \tilde{I}_{i}}^{L}}{h_{C \tilde{I}_{i}}^{U}}\left(c i_{2 i}^{U}+c i_{3 i}^{U}\right)+\right. \\
& \left.\frac{4 h_{C \tilde{I}_{i}}^{U}-3 h_{C \tilde{I}_{i}}^{L}}{h_{C \tilde{I}_{i}}^{U}}\left(c i_{1 i}^{U}+c i_{4 i}^{U}\right)\right] .
\end{aligned}
$$


The higher the likelihood-based compromise value $C V_{i}$, the better the alternative $z_{i}$ is. Thus, the $m$ alternatives can be ranked according to the decreasing order of $C V_{i}$ for all $z_{i} \in Z$. Subsequently, this paper chooses the best compromise alternative with the maximum value among all of the $C V_{i}$ values.

\subsection{Proposed Algorithm}

Using a likelihood-based compromise approach, the proposed MCDA method based on IT2 TrFNswithin the decision environment of IT2 FSs falls into four phases, including the phase of problem formulation, the phase of establishing the likelihood-based indices, the phase of determining the likelihood-based compromise values, and the phase of ranking the alternatives. Figure 2 shows the conceptual framework of the proposed method in detail.

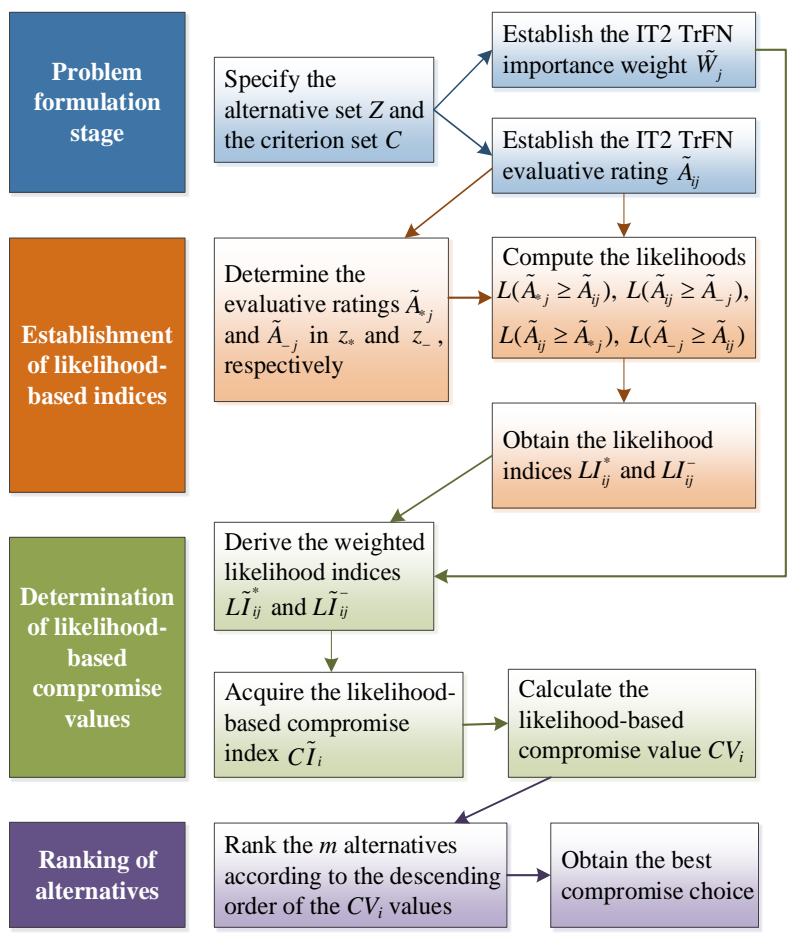

Fig 2: The conceptual framework of the proposed method.

The proposed MCDA method using a likelihood-based compromise approach can be summarized in the following steps:

\section{Steps 1 and 2: Problem formulation stage}

Step 1: Formulate an MCDA problem. Specify the alternative set $Z=\left\{z_{1}, z_{2}, \cdots, z_{m}\right\}$ and the criterion set $C=\left\{c_{1}, c_{2}, \cdots, c_{n}\right\}$, which is divided into $C_{\mathrm{I}}$ and $C_{\mathrm{II}}$.

Step 2: Establish the IT2 TrFN evaluative rating $\tilde{A}_{i j}$ in (9) for alternative $z_{i} \in Z$ with respect to criterion $c_{j} \in C$ and the IT2 TrFN importance weight $\tilde{W}_{j}$ in (11) for criterion $c_{j} \in C$.

\section{Steps 3-6: Establishment of likelihood-based indices}

Step 3: Acquire the evaluative rating $\tilde{A}_{* j}$ in (13) of the approximate positive-ideal solution $z_{*}$ with respect to criterion $c_{j} \in C$ using (14) and (15).

Step 4: Obtain the evaluative rating $\tilde{A}_{-j}$ in (17) of the approximate negative-ideal solution $z_{-}$with respect to criterion $c_{j} \in C$ using (18) and (19).

Step 5: Apply (21) to compute the lower likelihoods $L^{L}\left(\tilde{A}_{*_{j}} \geq \tilde{A}_{i j}\right) \quad$ and $\quad L^{L}\left(\tilde{A}_{i j} \geq \tilde{A}_{-j}\right) \quad$ for $\quad c_{j} \in C_{\mathrm{I}} \quad$ and $L^{L}\left(\tilde{A}_{i j} \geq \tilde{A}_{* j}\right)$ and $L^{L}\left(\tilde{A}_{-j} \geq \tilde{A}_{i j}\right)$ for $c_{j} \in C_{\mathrm{II}}$. Apply (22) to compute the upper likelihoods $L^{U}\left(\tilde{A}_{* j} \geq \tilde{A}_{i j}\right)$ and $L^{U}\left(\tilde{A}_{i j} \geq \tilde{A}_{-j}\right) \quad$ for $\quad c_{j} \in C_{\mathrm{I}} \quad$ and $\quad L^{U}\left(\tilde{A}_{i j} \geq \tilde{A}_{* j}\right) \quad$ and $L^{U}\left(\tilde{A}_{-j} \geq \tilde{A}_{i j}\right)$ for $c_{j} \in C_{\mathrm{II}}$.

Step 6: Use (23) and (24) to determine the likelihood index $L I_{i j}^{*}$ for each $z_{i} \in Z$ with respect to $c_{j} \in C$. Use (23) and (25) to determine the likelihood index $L I_{i j}^{-}$for each $z_{i} \in Z$ with respect to $c_{j} \in C$.

Steps 7-9: Determination of likelihood-based compromise values

Step 7: Derive the weighted likelihood indices $L \tilde{I}_{i j}^{*}$ in (26) and $L \tilde{I}_{i j}^{-}$in (27) using (28) and (29), respectively, for $z_{i} \in Z$ and $c_{j} \in C$.

Step 8: Employ (34) and (35) to establish the likelihoodbased compromise index $C \tilde{I}_{i}$ in (30) for $z_{i} \in Z$.

Step 9: Determine the likelihood-based compromise value $C V_{i}$ for $z_{i} \in Z$ using (36).

Step 10: Ranking of alternatives

Step 10: Rank the $m$ alternatives according to the descending order of the $C V_{i}$ values. The alternative with the largest $C V_{i}$ value is the best compromise choice.

\section{ILLUSTRATIVE APPLICATION}

This section illustrates and discusses the proposed MCDA methodby applying it to the car evaluation problempresented by Chen and Lee (2010b).

In the car evaluation problem, three decision makers use four criteria, i.e., safety $\left(c_{1}\right)$, price $\left(c_{2}\right)$, appearance $\left(c_{3}\right)$, and performance $\left(c_{4}\right)$, to evaluate three cars. The set of decision alternatives is denoted by $Z=\left\{z_{1}, z_{2}, z_{3}\right\}$. The set of evaluative criteria is denoted by $C=\left\{c_{1}, c_{2}, c_{3}, c_{4}\right\}$, where $C_{\mathrm{I}}=\left\{c_{1}, c_{3}, c_{4}\right\}$ and $C_{\mathrm{II}}=\left\{c_{2}\right\}$. Chen and Lee (2010b) combined the three decision makers' opinions to obtain an average weighting matrix and an average decision matrix. Based on the two average matrices, the evaluative ratings $\tilde{A}_{i j}$ of each $z_{i} \in Z$ with respect to $c_{j} \in C$ and the importance weights $\tilde{W}_{j}$ of each $c_{j} \in C$ are shown in Table 1. Note that we have already implemented the problem formulation stage (including Steps 1 and 2). 
Table 1: Data of the IT2 TrFN evaluative ratings and importance weights

\begin{tabular}{|l|l|l|}
\hline$z_{i}$ & $c_{j}$ & $\tilde{A}_{i j}$ \\
\hline$z_{1}$ & $c_{1}$ & {$[(0.67,0.77,0.77,0.85 ; 0.9),(0.57,0.77,0.77,0.93 ; 1.0)]$} \\
\hline & $c_{2}$ & {$[(0.85,0.93,0.93,0.97 ; 0.9),(0.77,0.93,0.93,1.00 ; 1.0)]$} \\
\hline & $c_{3}$ & {$[(0.85,0.93,0.93,0.97 ; 0.9),(0.77,0.93,0.93,1.00 ; 1.0)]$} \\
\hline & $c_{4}$ & {$[(0.85,0.93,0.93,0.97 ; 0.9),(0.77,0.93,0.93,1.00 ; 1.0)]$} \\
\hline$z_{2}$ & $c_{1}$ & {$[(0.73,0.83,0.83,0.90 ; 0.9),(0.63,0.83,0.83,0.97 ; 1.0)]$} \\
\hline & $c_{2}$ & {$[(0.78,0.87,0.87,0.92 ; 0.9),(0.70,0.87,0.87,0.97 ; 1.0)]$} \\
\hline & $c_{3}$ & {$[(0.90,0.97,0.97,0.98 ; 0.9),(0.83,0.97,0.97,1.00 ; 1.0)]$} \\
\hline & $c_{4}$ & {$[(0.90,0.97,0.97,0.98 ; 0.9),(0.83,0.97,0.97,1.00 ; 1.0)]$} \\
\hline & $c_{1}$ & {$[(0.78,0.87,0.87,0.92 ; 0.9),(0.70,0.87,0.87,0.97 ; 1.0)]$} \\
\hline & $c_{2}$ & {$[(0.90,0.97,0.97,0.98 ; 0.9),(0.83,0.97,0.97,1.00 ; 1.0)]$} \\
\hline & $c_{3}$ & {$[(0.53,0.63,0.63,0.73 ; 0.9),(0.43,0.63,0.63,0.83 ; 1.0)]$} \\
\hline & $c_{4}$ & {$[(0.85,0.93,0.93,0.97 ; 0.9),(0.77,0.93,0.93,1.00 ; 1.0)]$} \\
\hline & $c_{j}$ & $\tilde{W}_{j}$ \\
\hline & $c_{1}$ & {$[(0.90,0.97,0.97,0.98 ; 0.9),(0.83,0.97,0.97,1.00 ; 1.0)]$} \\
\hline & $c_{2}$ & {$[(0.90,0.97,0.97,0.98 ; 0.9),(0.83,0.97,0.97,1.00 ; 1.0)]$} \\
\hline & $c_{3}$ & {$[(0.53,0.63,0.63,0.73 ; 0.9),(0.43,0.63,0.63,0.83 ; 1.0)]$} \\
\hline & $c_{4}$ & {$[(0.85,0.93,0.93,0.97 ; 0.9),(0.77,0.93,0.93,1.00 ; 1.0)]$} \\
\hline
\end{tabular}

The remaining steps of the proposed MCDA method using a likelihood-based compromise approach are illustrated as follows. Based on Step 3 in the establishment stage of likelihood-based indices, the IT2 TrFN evaluative ratings of the approximate positive-ideal solution $Z_{*}$ with respect to criterion $c_{j} \in C$ were acquired as follows:

$$
\begin{gathered}
\tilde{A}_{*_{1}}=[(0.78,0.87,0.87,0.92 ; 0.9),(0.70,0.87,0.87,0.97 ; \\
1.0)], \\
\tilde{A}_{*_{2}}=[(0.78,0.87,0.87,0.92 ; 0.9),(0.70,0.87,0.87,0.97 ; \\
1.0)], \\
\tilde{A}_{*_{3}}=[(0.90,0.97,0.97,0.98 ; 0.9),(0.83,0.97,0.97,1.00 ; \\
1.0)], \\
\tilde{A}_{*_{4}}=[(0.90,0.97,0.97,0.98 ; 0.9),(0.83,0.97,0.97,1.00 ; \\
1.0)] .
\end{gathered}
$$

In Step 4, the IT2 TrFN evaluative ratings of the approximate negative-ideal solution $z_{-}$with respect to criterion $c_{j} \in C$ were obtained as follows:

$$
\begin{gathered}
\tilde{A}_{-1}=[(0.73,0.83,0.83,0.90 ; 0.9),(0.63,0.83,0.83,0.97 \\
1.0)], \\
\tilde{A}_{-2}=[(0.90,0.97,0.97,0.98 ; 0.9),(0.83,0.97,0.97,1.00 ; \\
1.0)], \\
\tilde{A}_{-3}=[(0.53,0.63,0.63,0.73 ; 0.9),(0.43,0.63,0.63,0.83 ; \\
1.0)], \\
\tilde{A}_{-4}=[(0.85,0.93,0.93,0.97 ; 0.9),(0.77,0.93,0.93,1.00 \\
1.0)]
\end{gathered}
$$

In Step 5, this study calculated $L^{L}\left(\tilde{A}_{*_{j}} \geq \tilde{A}_{i j}\right)$, $L^{U}\left(\tilde{A}_{* j} \geq \tilde{A}_{i j}\right), L^{L}\left(\tilde{A}_{i j} \geq \tilde{A}_{-j}\right)$, and $L^{U}\left(\tilde{A}_{i j} \geq \tilde{A}_{-j}\right)$ of each $z_{i} \in Z$ with respect to $c_{j} \in C_{\mathrm{I}}$ and $L^{L}\left(\tilde{A}_{i j} \geq \tilde{A}_{* j}\right)$, $L^{U}\left(\tilde{A}_{i j} \geq \tilde{A}_{* j}\right), L^{L}\left(\tilde{A}_{-j} \geq \tilde{A}_{i j}\right)$, and $L^{U}\left(\tilde{A}_{-j} \geq \tilde{A}_{i j}\right)$ of each $z_{i} \in Z$ with respect to $c_{j} \in C_{\mathrm{II}}$. The computed results are presented in Table 2.

Table 2: Results of the lower and upper likelihoods

\begin{tabular}{|l|l|l|l|l|l|}
\hline$z_{i}$ & $c_{j}\left(\in C_{\mathrm{I}}\right)$ & $L^{L}\left(\tilde{A}_{* j} \geq \tilde{A}_{i j}\right)$ & $L^{U}\left(\tilde{A}_{*_{j}} \geq \tilde{A}_{i j}\right)$ & $L^{L}\left(\tilde{A}_{i j} \geq \tilde{A}_{-j}\right)$ & $L^{U}\left(\tilde{A}_{i j} \geq \tilde{A}_{-j}\right)$ \\
\hline$z_{1}$ & $c_{1}$ & 0.6786 & 0.8500 & 0.2600 & 0.4135 \\
\hline & $c_{3}$ & 0.5676 & 0.7419 & 0.9043 & 1.0000 \\
\hline & $c_{4}$ & 0.5676 & 0.7419 & 0.4242 & 0.5758 \\
\hline$z_{2}$ & $c_{1}$ & 0.5417 & 0.7195 & 0.4205 & 0.5795 \\
\hline & $c_{3}$ & 0.4074 & 0.5926 & 0.9343 & 1.0000 \\
\hline & $c_{4}$ & 0.4074 & 0.5926 & 0.5676 & 0.7419 \\
\hline$z_{3}$ & $c_{1}$ & 0.4054 & 0.5946 & 0.5417 & 0.7195 \\
\hline & $c_{3}$ & 0.9343 & 0.7419 & 0.4000 & 0.6000 \\
\hline & $c_{4}$ & 0.5676 & 0.4242 & 0.5758 \\
\hline$z_{i}$ & $c_{j}\left(\in C_{\mathrm{II}}\right)$ & $L^{L}\left(\tilde{A}_{i j} \geq \tilde{A}_{* j}\right)$ & $L^{U}\left(\tilde{A}_{i j} \geq \tilde{A}_{*_{j}}\right)$ & $L^{L}\left(\tilde{A}_{-j} \geq \tilde{A}_{i j}\right)$ & $L^{U}\left(\tilde{A}_{-j} \geq \tilde{A}_{i j}\right)$ \\
\hline
\end{tabular}




\begin{tabular}{|l|l|l|l|l|l|}
\hline$z_{1}$ & $c_{2}$ & 0.6279 & 0.7949 & 0.5676 & 0.7419 \\
\hline$z_{2}$ & $c_{2}$ & 0.4054 & 0.5946 & 0.7188 & 0.8929 \\
\hline$z_{3}$ & $c_{2}$ & 0.7188 & 0.8929 & 0.4074 & 0.5926 \\
\hline
\end{tabular}

In Step 6, the calculated results of the likelihood indices $L I_{i j}^{*}$ and $L I_{i j}^{-}$for each $z_{i} \in Z$ with respect to $c_{j} \in C$ are shown in Table 3

Table 3: Results of the likelihood indices

\begin{tabular}{|l|l|l|l|l|l|l|}
\hline$c_{j}$ & $L I_{1 j}^{*}$ & $L I_{2 j}^{*}$ & $L I_{3 j}^{*}$ & $L I_{1 j}^{-}$ & $L I_{2 j}^{-}$ & $L I_{3 j}^{-}$ \\
\hline$c_{1}$ & 0.7643 & 0.6306 & 0.5000 & 0.3367 & 0.5000 & 0.6306 \\
\hline$c_{2}$ & 0.7114 & 0.5000 & 0.8058 & 0.6548 & 0.8058 & 0.5000 \\
\hline$c_{3}$ & 0.6548 & 0.5000 & 0.9672 & 0.9521 & 0.9672 & 0.5000 \\
\hline$c_{4}$ & 0.6548 & 0.5000 & 0.6548 & 0.5000 & 0.6548 & 0.5000 \\
\hline
\end{tabular}

Based on Step 7 in the determination stage of likelihoodbased compromise values, the weighted likelihood indices $L \tilde{I}_{i j}^{*}$ and $L \tilde{I}_{i j}^{-}$were derived for $z_{i} \in Z$ and $c_{j} \in C$, and the results of the computation are depicted in Table 4.

Table 4: Results of the weighted likelihood indices

\begin{tabular}{|c|c|c|}
\hline$z_{i}$ & $c_{j}$ & $L_{i j}^{*}$ \\
\hline$z_{1}$ & $c_{1}$ & {$[(0.6879,0.7414,0.7414,0.7490 ; 0.9),(0.6344,0.7414,0.7414,0.7643 ; 1.0)]$} \\
\hline & $c_{2}$ & {$[(0.6403,0.6901,0.6901,0.6972 ; 0.9),(0.5905,0.6901,0.6901,0.7114 ; 1.0)]$} \\
\hline & $c_{3}$ & {$[(0.3470,0.4125,0.4125,0.4780 ; 0.9),(0.2816,0.4125,0.4125,0.5435 ; 1.0)]$} \\
\hline & $c_{4}$ & {$[(0.5566,0.6090,0.6090,0.6352 ; 0.9),(0.5042,0.6090,0.6090,0.6548 ; 1.0)]$} \\
\hline$z_{2}$ & $c_{1}$ & {$[(0.5675,0.6117,0.6117,0.6180 ; 0.9),(0.5234,0.6117,0.6117,0.6306 ; 1.0)]$} \\
\hline & $c_{2}$ & {$[(0.4500,0.4850,0.4850,0.4900 ; 0.9),(0.4150,0.4850,0.4850,0.5000 ; 1.0)]$} \\
\hline & $c_{3}$ & {$[(0.2650,0.3150,0.3150,0.3650 ; 0.9),(0.2150,0.3150,0.3150,0.4150 ; 1.0)]$} \\
\hline & $c_{4}$ & {$[(0.4250,0.4650,0.4650,0.4850 ; 0.9),(0.3850,0.4650,0.4650,0.5000 ; 1.0)]$} \\
\hline$z_{3}$ & $c_{1}$ & {$[(0.4500,0.4850,0.4850,0.4900 ; 0.9),(0.4150,0.4850,0.4850,0.5000 ; 1.0)]$} \\
\hline & $c_{2}$ & {$[(0.7252,0.7816,0.7816,0.7897 ; 0.9),(0.6688,0.7816,0.7816,0.8058 ; 1.0)]$} \\
\hline & $c_{3}$ & {$[(0.5126,0.6093,0.6093,0.7061 ; 0.9),(0.4159,0.6093,0.6093,0.8028 ; 1.0)]$} \\
\hline & $c_{4}$ & {$[(0.5566,0.6090,0.6090,0.6352 ; 0.9),(0.5042,0.6090,0.6090,0.6548 ; 1.0)]$} \\
\hline$z_{i}$ & $c_{j}$ & $L \tilde{I}_{i j}^{-}$ \\
\hline$z_{1}$ & $c_{1}$ & {$[(0.3030,0.3266,0.3266,0.3300 ; 0.9),(0.2795,0.3266,0.3266,0.3367 ; 1.0)]$} \\
\hline & $c_{2}$ & {$[(0.5893,0.6352,0.6352,0.6417 ; 0.9),(0.5435,0.6352,0.6352,0.6548 ; 1.0)]$} \\
\hline & $c_{3}$ & {$[(0.5046,0.5998,0.5998,0.6950 ; 0.9),(0.4094,0.5998,0.5998,0.7902 ; 1.0)]$} \\
\hline & $c_{4}$ & {$[(0.4250,0.4650,0.4650,0.4850 ; 0.9),(0.3850,0.4650,0.4650,0.5000 ; 1.0)]$} \\
\hline$z_{2}$ & $c_{1}$ & {$[(0.4500,0.4850,0.4850,0.4900 ; 0.9),(0.4150,0.4850,0.4850,0.5000 ; 1.0)]$} \\
\hline & $c_{2}$ & {$[(0.7252,0.7816,0.7816,0.7897 ; 0.9),(0.6688,0.7816,0.7816,0.8058 ; 1.0)]$} \\
\hline & $c_{3}$ & {$[(0.5126,0.6093,0.6093,0.7061 ; 0.9),(0.4159,0.6093,0.6093,0.8028 ; 1.0)]$} \\
\hline & $c_{4}$ & {$[(0.5566,0.6090,0.6090,0.6352 ; 0.9),(0.5042,0.6090,0.6090,0.6548 ; 1.0)]$} \\
\hline$z_{3}$ & $c_{1}$ & {$[(0.5675,0.6117,0.6117,0.6180 ; 0.9),(0.5234,0.6117,0.6117,0.6306 ; 1.0)]$} \\
\hline & $c_{2}$ & {$[(0.4500,0.4850,0.4850,0.4900 ; 0.9),(0.4150,0.4850,0.4850,0.5000 ; 1.0)]$} \\
\hline & $c_{3}$ & {$[(0.2650,0.3150,0.3150,0.3650 ; 0.9),(0.2150,0.3150,0.3150,0.4150 ; 1.0)]$} \\
\hline & $c_{4}$ & {$[(0.4250,0.4650,0.4650,0.4850 ; 0.9),(0.3850,0.4650,0.4650,0.5000 ; 1.0)]$} \\
\hline & & \\
\hline
\end{tabular}

In Step 8, the likelihood-based compromise indices were determined as follows:

$C \tilde{I}_{1}=[(0.3867,0.4524,0.4524,0.5308 ; 0.9), \quad(0.3264$, $0.4524,0.4524,0.6289 ; 1.0)]$,

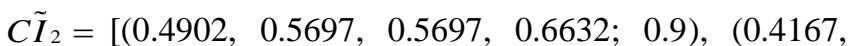
$0.5697,0.5697,0.7801 ; 1.0)]$,

$C \tilde{I}_{3}=[(0.3729,0.4303,0.4303,0.4954 ; 0.9), \quad(0.3199$, $0.4303,0.4303,0.5775 ; 1.0)]$. 
Next, in Step 9, the corresponding likelihood-based compromise values were calculated as follows: $C V_{1}=$ $0.9146, C V_{2}=1.1505$, and $C V_{3}=0.8675$.

Based on Step 10 in the final ranking stage, the ranking order of the three cars is $z_{2} \succ z_{1} \succ z_{3}$ because $C V_{2}>C V_{1}>C V_{3}$. Therefore, the best compromise choice is the alternative $z_{2}$. This result is the same as that obtained using the method of Chen and Lee (2010b), which is based on the ranking values and the arithmetic operations of interval type-2 fuzzy sets. Thus, the feasibility and applicability of the proposed method was validated by the analysis of the car evaluation problem.

\section{CONCLUSION}

Uncertain and imprecise information often occurs in realworld MCDA problems. The theory of IT2 FSs, IT2 TrFNs especially, is useful and convenient for modeling impressions and quantifying the ambiguous nature of subjective judgments. Using the likelihood-based compromise approach, this paper developed a new decisionmaking method designed to address MCDA problems based on IT2 TrFNswithin the decision environment of IT2 FSs. To facilitate anchored judgments in the decision-making process, the proposed method presented the concept of the approximate positive-ideal and negative-ideal solutions that are generated from the characteristics of the alternatives. Next, the likelihood indices and the weighted likelihood indices were established based on the likelihood of an IT2 TrFN binary relation between the alternatives and the approximate ideals. In general, processing sophisticated IT2 TrFN data is troublesome in practice. Nevertheless, this paper provided a simple and effective method based on the extended concept of likelihoods for handling complicated IT2 TrFN evaluative ratings and importance weights in the likelihood indices and the weighted likelihood indices, respectively. Additionally, the proposed method presented the novel concept of the likelihood-based compromise indices and the likelihood-based compromise values for the purpose of ranking the priority order of the alternatives. The practical applicability of the proposed method was validated via applications to the car evaluation problem. Comparisons of the results also demonstrated the effectiveness of the proposed method. Therefore, the proposed method is useful and valuable for addressing MCDA problems in the decision context of IT2 TrFNs.

\section{ACKNOWLEDGEMENT}

The authors are grateful for grant funding support from the Taiwan Ministry of Science and Technology (MOST 1052410-H-182-007-MY3) and Chang Gung Memorial Hospital (BMRP 574 and CMRPD2F0201) during the completion of this study.

\section{CONFLICT OF INTEREST STATEMENT}

The authors declare that there is no conflict of interests regarding the publication of this paper.

\section{REFERENCES}

[1]. E. Celik, A. Taskin Gumus, An outranking approach based on interval type-2 fuzzy sets to evaluate preparedness and response ability of non-governmental humanitarian relief organizations, Computers and Industrial Engineering 101 (2016) 21-34.

[2]. T.-Y. Chen, A signed-distance-based approach to importance assessment and multi-criteria group decision analysis based on interval type-2 fuzzy set, Knowledge and Information Systems 35 (1) (2013) 193-231.

[3]. T.-Y. Chen, An ELECTRE-based outranking method formultiple criteria group decision making using interval type-2 fuzzy sets, Information Sciences 263 (2014a) 1-21.

[4]. T.-Y. Chen, A PROMETHEE-based outranking method formultiple criteria decision analysis with interval type-2 fuzzy sets, Soft Computing 18 (5) (2014b) 923-940.

[5]. T.-Y. Chen, Multiple criteria decision analysis using prioritised interval type-2 fuzzy aggregation operators and its application to site selection, Technological and Economic Development of Economy 23 (1) (2017) 1-21.

[6]. T.-Y. Chen, C.-H. Chang, J.-f.R.Lu, The extended QUALIFLEX method for multiple criteria decision analysis based on interval type-2 fuzzy setsand applications to medical decision making, European Journal of Operational Research 226 (3) (2013) 615-625.

[7]. S.-M. Chen, L.-W. Lee, Fuzzy decision-making based on likelihood-based comparison relations, IEEE Transactions on Fuzzy Systems 18 (3) (2010a) 613-628.

[8]. S.-M. Chen, L.-W. Lee, Fuzzy multiple attributes group decision-making based on the ranking values and the arithmetic operations of interval type-2 fuzzy sets, Expert Systems with Applications 37 (1) (2010b) 824-833.

[9]. S.-M. Chen, L.-W. Lee, Fuzzy multiple criteria hierarchical group decision-making based on interval type-2 fuzzy sets, IEEE Transactions on Systems, Man, and Cybernetics-Part A: Systems and Humans 40 (5) (2010c) 1120-1128.

[10]. H.-L. Lai, T.-Y. Chen, Client acceptance method for audit firms based on interval-valued fuzzy numbers, Technological and Economic Development of Economy 21 (1) (2015) 1-27.

[11]. D.-F. Li, Linear programming method for MADM with interval-valued intuitionistic fuzzy sets, Expert Systems with Applications 37 (8) (2010a) 5939-5945.

[12]. D.-F. Li, TOPSIS-based nonlinear-programming methodology for multiattribute decision making with interval-valued intuitionistic fuzzy sets, IEEE Transactions on Fuzzy Systems 18 (2) (2010b) 299-311.

[13]. D.-F. Li, Closeness coefficient based nonlinear programming method for interval-valued intuitionistic fuzzy multiattribute decision making with incomplete preference information, Applied Soft Computing 11 (4) (2011) 34023418.

[14]. J. M. Mendel, Uncertain Rule-Based Fuzzy Logic Systems: Introduction and New Directions, Prentice-Hall, Upper-Saddle River, NJ, 2001.

[15]. J.M. Mendel, Type-2 fuzzy sets and systems: an overview, IEEE Computational Intelligence Magazine 2 (1) (2007) 20-29.

[16]. J.M. Mendel, Type-2 fuzzy sets-a tribal parody, IEEE 
Computational Intelligence Magazine 5 (4) (2010) 24-27.

[17]. J.M. Mendel, R.I.B. John, Type-2 fuzzy sets made simple. IEEE Transactions on Fuzzy Systems 10 (2) (2002) 117-127.

[18]. M. Moharrer, H. Tahayori, L. Livi, A. Sadeghian, A. Rizzi, Interval type-2 fuzzy sets to model linguistic label perception in online services satisfaction, Soft Computing 19 (1) (2015) 237-250.

[19]. J. Qin, X. Liu, W. Pedrycz, An extended TODIM multi-criteria group decision making method for green supplier selection in interval type-2 fuzzy environment, European Journal of Operational Research 258 (2) (2017) 626-638.

[20]. K. Siminski, Interval type-2 neuro-fuzzy system with implication-based inference mechanism, Expert Systems with Applications 79 (2017) 140-152.

[21]. S. Singh, H. Garg, Distance measures between type-2 intuitionistic fuzzy sets and their application to multicriteria decision-making process, Applied Intelligence 46 (4) (2017) 788-799.

[22]. J.-C. Wang, T.-Y. Chen, A closeness coefficient-based multiple criteria decision-making method using interval type-2 fuzzy sets and its application to watershed site selection, Journal of Industrial and Production Engineering 31 (1) (2014) 1-16.

[23]. J.-C. Wang, T.-Y. Chen, A simulated annealing-based permutation method and experimental analysis for multiple criteria decision analysis with interval type-2 fuzzy sets, Applied Soft Computing 36 (2015) 57-69.

[24]. J.-C. Wang, C.-Y. Tsao, T.-Y. Chen, A likelihoodbased QUALIFLEX method with interval type-2 fuzzy sets for multiple criteria decision analysis, Soft Computing19 (8) (2015) 2225-2243.

[25]. D. Wu, J.M. Mendel, Uncertainty measures for interval type-2 fuzzy sets, Information Sciences 177 (23) (2007) 5378-5393.

[26]. L.A. Zadeh, The concept of a linguistic variable and its application to approximate reasoning-I,Information Sciences 8 (3) (1975) 199-249.

[27]. Z. Zhang, S. Zhang, A novel approach to multi attribute group decision making based on trapezoidal interval type-2 fuzzy soft sets, Applied Mathematical Modelling 37 (7) (2013) 4948-4971.

[28]. T. Zhao, J. Xiao, General type-2 fuzzy rough sets based on $\alpha$-plane Representation theory, Soft Computing 18 (2) (2014) 227-237.

[29]. T. Zhao, J. Xiao, Y. Li, X. Deng, A new approach to similarity and inclusion measures between general type-2 fuzzy sets, Soft Computing 18 (4) (2014) 809-823.

[30]. L. Zhong, L. Yao, An ELECTRE I-based multi-criteria group decision making method with interval type-2 fuzzy numbers and its application to supplier selection, Applied Soft Computing 57 (2017) 556-576.

[31]. L. Zhou, H. Li, K. Sun, Teaching performance evaluation by means of a hierarchical multifactorial evaluation model based on type-2 fuzzy sets, Applied Intelligence 46 (1) (2017) 34-44. 